University of Warwick institutional repository: http://go.warwick.ac.uk/wrap This paper is made available online in accordance with publisher policies. Please scroll down to view the document itself. Please refer to the repository record for this item and our policy information available from the repository home page for further information.

To see the final version of this paper please visit the publisher's website. Access to the published version may require a subscription.

Author(s): Andrew D. Millard and Nicholas H. Mann Article Title: A temporal and spatial investigation of cyanophage abundance in the Gulf of Aqaba Red Sea Year of publication: 2006 Link to published version: http://dx.doi.org/ 10.1017/S0025315406013415

Publisher statement: None 


\title{
A temporal and spatial investigation of cyanophage abundance in the Gulf of Aqaba, Red Sea
}

\author{
Andrew D. Millard* ${ }^{\dagger}$ and Nicholas H. Mann* \\ *Department of Biological Sciences, University of Warwick, Coventry, CV4 7AL, UK. \\ ${ }^{\dagger}$ Corresponding author, e-mail: a.d.millard@warwick.ac.uk
}

\begin{abstract}
The aim of this study was to determine the abundance of cyanophages over an annual cycle in the Red Sea from the period April 1999 to December 1999 at a range of depths. Cyanophage numbers from 71 water samples were determined by the use of plaque assays using four different Synechococcus strains. The results indicate that cyanophage are found throughout the water column from surface waters to depths of $150 \mathrm{~m}$, with a discrete maximum in the number of cyanophages in the summer months of July, August and September at a depth of $30 \mathrm{~m}$. Eighty-seven cyanophages were isolated and characterized in terms of host range, genome size and possession of a myoviral portal vertex gene. Cyanophages were found to infect multiple strains of Synechococcus from different phylogenetic clades. The genome sizes of cyanophages were also found to be bigger than previously estimated.
\end{abstract}

\section{INTRODUCTION}

Cyanophages are bacteriophages capable of infecting cyanobacteria. They are ubiquitous in the marine environment and have been isolated from a number of diverse locations that include the English Channel, Sargasso Sea and the Gulf of Mexico (Suttle \& Chan, 1993; Waterbury \& Valois, 1993; Wilson et al., 1993; Suttle \& Chan, 1994; Sullivan et al., 2003). Cyanophages infecting marine Synechococcus strains have been the object of study for over ten years and more recently cyanophages infecting Prochlorococcus have also been isolated and characterized (Sullivan et al., 2003). The influence that cyanophages can have on marine ecosystems may be broader than previously thought with the recent discovery that cyanophages contain genes that encode proteins essential for photosynthesis, suggesting they may influence the photosynthetic ability of infected hosts (Mann et al., 2003).

Previous studies have shown that cyanophage titres change on a seasonal basis (Waterbury \& Valois, 1993). Whilst others have found that cyanophage diversity changes on a spatial scale (Frederickson et al., 2003). This study aimed to investigate the abundance of cyanophages both temporally and spatially in the Gulf of Aqaba in the Red Sea. The Gulf of Aqaba is separated from the Straits of Tiran by a shallow sill at a depth of $250 \mathrm{~m}$, which restricts the flow of Red Sea water into the Gulf. This causes a long residence time for water in the Gulf, with the surface water resident for one year and deeper waters between 3 and 8 years (Reiss \& Hottinger, 1984), and combined with a lack of drainage into the straits results in a body of water that although coastal in geographical terms is representative of open ocean water in terms of its nutrient status (Klinker et al., 1976; Wolfvecht et al., 1992). There is a distinct seasonal pattern to the circulation of water in the Gulf, which is characterized by stratification of the water column in the summer months, which disappears and by winter the water column is mixed as nutrient-rich water has risen to the surface (Klinker et al., 1976; Wolfvecht et al., 1992). The Synechococcus and Prochlorococcus community within the Gulf of Aqaba has previously been studied in detail (Fuller et al., 2003, 2005). The diversity of the Synechococcus in the Red Sea has previously been found to co-vary with cyanophage diversity indicating that cyanophage diversity in the Red Sea can have a controlling influence on Synechococcus diversity (Mühling et al., 2005).

\section{MATERIALS AND METHODS}

Host strains and media

The host Synechococcus strains used in this study were maintained in artificial seawater (ASW) medium (Wyman et al., 1985) in $100 \mathrm{ml}$ batch cultures in $250 \mathrm{ml}$ conical flasks under constant illumination (5$36 \mu$ moles $\mathrm{m}^{-2} \mathrm{~s}^{-1}$ ) at $25^{\circ} \mathrm{C}$. Larger volumes were grown in litre and 2-1 vessels to which $0.5 \mathrm{~g} \mathrm{l}^{-1}$ of $\mathrm{NaHCO}_{3}$ was added. Cultures were aerated and stirred constantly. The Synechococcus strains used in this study were WH7803, WH8103, RS9906 and RS9911. These four strains were representatives of four different phylogenetic clades as determined by 16S rRNA sequence analysis (Fuller et al., 2003). Synechococcus sp. WH7803 and WH8013 are both well characterized strains and have been in culture for over 20 years. Synechococcus sp. RS9906 and RS9911 were both isolated by N. Fuller from the Gulf of Aqaba at the same time as water samples were collected during the period February 1999 to January 2000 (Fuller et al., 2003). Prochlorococcus sp. SS120 was cultured in the enriched seawater medium PCRS-11 (Partensky et al., 1999) in 100 $\mathrm{ml}$ volumes in $175 \mathrm{ml}$ volume Nunclon plastic flasks (Nalgene Nunc International, Rochester, NY ) or in $5 \mathrm{ml}$ 
volumes in plastic bijoux at $21^{\circ} \mathrm{C}$ under constant illumination $\left(5-36 \mu\right.$ moles $\left.\mathrm{m}^{-2} \mathrm{~s}^{-1}\right)$ without stirring.

\section{Location and sampling}

A total of 71 water samples was kindly collected over a nine-month period by N. Fuller from the Gulf of Aqaba at Station A $\left(29^{\circ} 28^{\prime} \mathrm{N} 34^{\circ} 55^{\prime} \mathrm{E}\right)$ between April 1999 and December 1999. Only single water samples were collected for each sampling point which varied in depth from surface waters down to $150 \mathrm{~m}$. Water was first passed through a Supor-450 filter $(0.45-\mu \mathrm{m}$ pore size, Gelman Sciences, Inc., Ann Arbor, Michigan) under a vacuum of $10 \mathrm{~mm} \mathrm{Hg}$. Water samples were then passed through a filter of pore size $0.20 \mu \mathrm{m}$ (Sartorius, Epsom, UK ) by the use of a syringe and stored in the dark at $4^{\circ} \mathrm{C}$ until ready for use. Samples were collected at depths of 10, 30, 50, 80, 100 and $150 \mathrm{~m}$ on the $19 \& 27$ April, 4 June, 18 July, 23 August, 7 September, 4 \& 18 October, 22 November and 27 December 1999. Additional samples were collected from $60 \mathrm{~m}$ on the $19 \& 27$ April and 14 June 1999; $125 \mathrm{~m}$ on 19 April, 14 June and 18 October 1999. On 11 May 1999 from depths of $0,50,80,100$ and $150 \mathrm{~m}$.

\section{Estimation of cyanophage abundance}

Cyanophage abundance was elucidated from these water samples by the use of plaque assays (Wilson et al., 1993). A minimum of three replicates were used in the enumeration of cyanophage numbers for all 71 water samples. All four Synechococcus strains were used to enumerate cyanophage numbers. The number, size and morphology of the plaques obtained were recorded.

\section{Isolation of cyanophages}

Cyanophages were isolated by the removal of a plaque with a Pasteur pipette and resuspension in $1 \mathrm{ml}$ of ASW. This phage preparation was then used in a further round of single plaques isolation, using serial dilutions if necessary. This process was repeated a total of three times to ensure that clonal isolates were obtained. Isolates were named following the previously suggested convention of Suttle (Suttle, 2000), with the letters RS used to designate the location as the Red Sea.

\section{Determination of cyanophage host range}

Host ranges were determined for the clonal stocks of cyanophage isolated from the Red Sea. Cyanophages were tested for infectivity against all four strains of Synechococcus and Prochlorococcus sp. SS120. Infection of Synechococcus sp. WH7803, WH8103 and RS9906 was tested by means of plaque assays; infection of Synechococcus sp. RS9911 was tested by means of well assays (Suttle \& Chan, 1993). Well assays were used for RS9911 as it is easier to culture this strain in liquid than on solid agar plates. For testing infection of Prochlorococcus, the phage lysate was added to triplicate samples of $5 \mathrm{ml}$ of exponentially growing culture. Phages S-WHM1 and S-PM2, previously isolated from Woods Hole and Plymouth respectively (Wilson et al., 1993), were used as negative controls as they had been shown not to infect Prochlorococcus sp. SS120 (Sullivan et al., 2003).

\section{$D \mathcal{N A}$ extraction and PCR amplification}

DNA was extracted from cyanophage preparations obtained by the propagation of cyanophages to produce confluent lysis of lawns of Synechococcus. The top layer of $0.4 \%$ agar from five or more plates was pooled and resuspended in $2 \mathrm{ml}$ of ASW, this was then centrifuged at $13,000 \times g$ to pellet the agar. DNA was then extracted from the supernatant (Wilson et al., 1993); this briefly comprised a phenol extraction, followed by a phenol/ chloroform $(1: 1)$ and an iso-amyl alcohol/chloroform $(1: 24)$ extraction. DNA was then precipitated by the addition of ammonium acetate and propan-2-ol, before being washed with $70 \%$ ethanol and resuspended in $50 \mu \mathrm{l} \mathrm{H}_{2} \mathrm{O}$.

Polymerase chain reaction (PCR) using the extracted DNA as a template was employed to screen phage isolates in order to aid in classification of the phages. The cyanomyovirus-specific g20 primers CPS-1 and CPS-2 were employed in a PCR using previously described conditions (Fuller et al., 1998) and the products were analysed on a $1 \%$ agarose gel. The presence of a PCR product of the correct size indicated that the phage was a member of the family Myoviridae. Phages that could not be identified as myoviruses by the use of PCR were examined by transmission electron microscopy (TEM) in order to characterize their morphology.

\section{Transmission electron microscopy}

Samples from cyanophage stocks were examined in a Joel Jem-100s TEM. Grids were first coated in butvar film (Sigma); a microscope slide was immersed in a solution of $1.5 \%(\mathrm{w} / \mathrm{v})$ butvar/chloroform for $30 \mathrm{~s}$, then removed and allowed to dry in the vapour of the chloroform. Several 200 mesh copper grids (Agar Scientific, Essex, UK) were placed shiny face down onto the butvar film and dried in an oven at $50^{\circ} \mathrm{C}$ for one hour. Fifty $\mu$ l of phage lysate was placed onto a butvar coated copper grid and left to absorb for $20 \mathrm{~min}$. The liquid was then carefully removed with blotting paper and $20 \mu \mathrm{l}$ of $2 \%(\mathrm{w} / \mathrm{v})$ uranyl acetate was added to the grid and immediately removed. A further $20 \mu \mathrm{l}$ of uranyl acetate $2 \%(\mathrm{w} / \mathrm{v})$ was added and left for $20 \mathrm{~s}$ before being removed. Grids were examined at an accelerating voltage of $80 \mathrm{kV}$ and images were obtained at magnifications of $\times 40,000-100,000$. The addition of $0.012 \mu \mathrm{m}$ diameter polystyrene beads (Agar Scientific, Essex, UK) served as a control in the measurement of phage particles.

\section{Determination of genome size}

The genome size of cyanophage isolates was determined by the use of pulsed-field gel electrophoresis (PFGE). High titre suspensions of cyanophage isolates were produced by the removal of the top layer of $0.4 \%(\mathrm{w} / \mathrm{v})$ agar from a plaque assay where confluent lysis had occurred. The top layer of $0.4 \%(\mathrm{w} / \mathrm{v})$ agar was resuspended in $1 \mathrm{ml}$ of ASW, left overnight at $4^{\circ} \mathrm{C}$ to allow phage diffusion, and then centrifuged at $13,000 \times g$ for 5 min to pellet the agar. The supernatant was removed and used to make agarose/phage plugs by using $50 \mu \mathrm{l}$ of phage lysate added to $50 \mu \mathrm{l}$ of molten 2\% low melting point agarose (Sigma) allowing it to set in plug moulds (Bio-Rad). Once set, the plugs were 

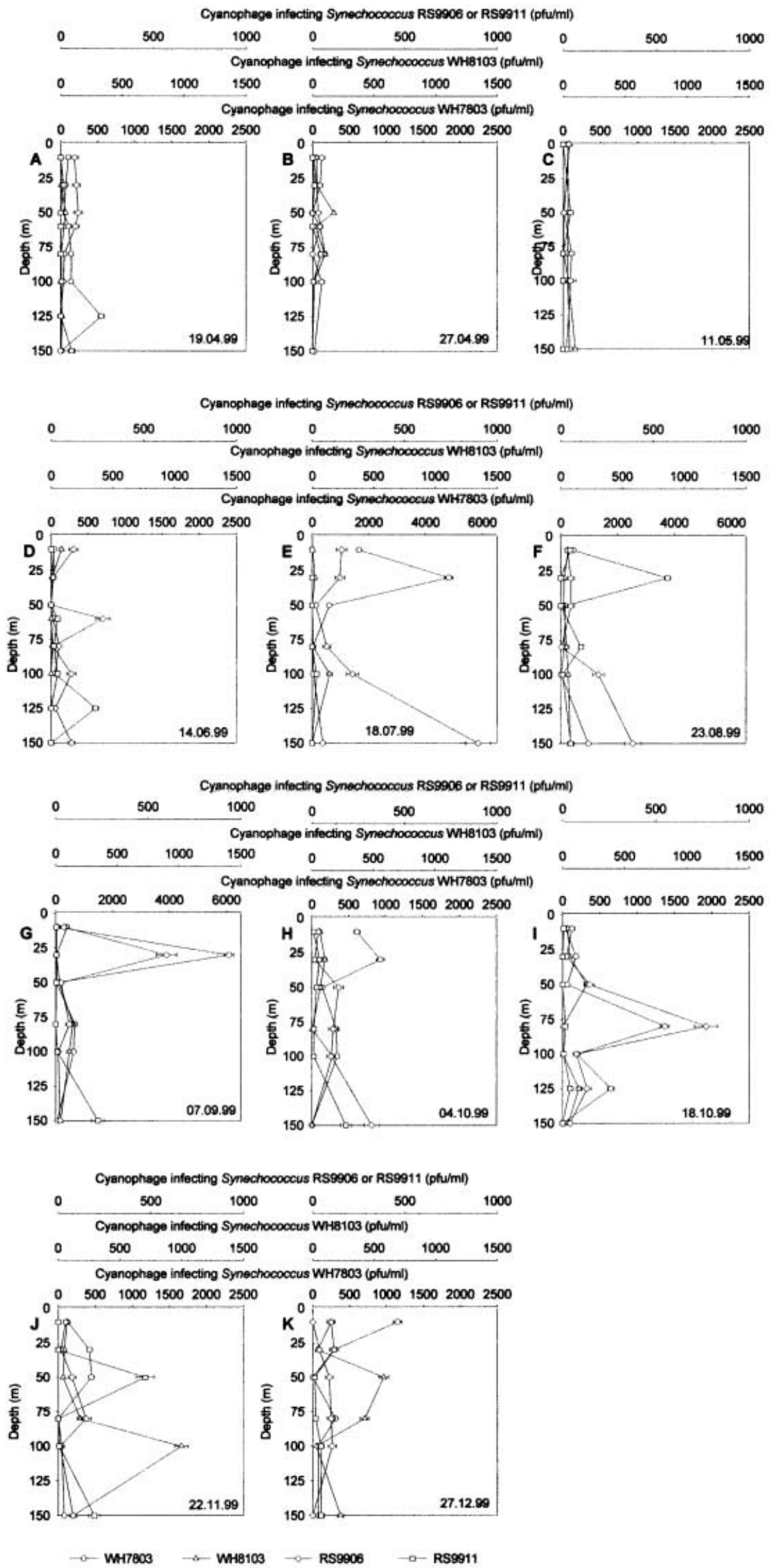

Figure 1. Depth profiles of the distribution of cyanophages (pfu/ml) in the Gulf of Aqaba from 19 April 1999 to 12 December 1999 from water samples collected from 0 to $150 \mathrm{~m}$ in depth. Cyanophage abundance was determined by the use of plaque assays

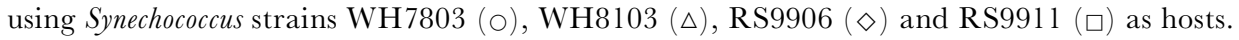


Table 1. Characterization of cyanophages from the Red Sea. Cyanophages were named using the nomenclature suggested by Suttle (2000); S (Synechococcus), RS (Red Sea), M (Myovirus) and 3 (isolate number). The date and depth the water sample used for isolation was collected from. PCR using cyanomyovirus specific primers CPS-1 and CPS-2 was used to identify the phages as cyanomyoviruses. Genome size was determined using PFGE. The host range of the cyanophages against Synechococcus sp. WH7803, WH8103, RS9906 was determined by plaque assays and well assays against RS9911 and Prochlorococcus SS120.

\begin{tabular}{|c|c|c|c|c|c|c|c|c|c|}
\hline Phage & $\begin{array}{l}\text { Date of } \\
\text { isolation }\end{array}$ & Depth & $\begin{array}{l}\text { Genome } \\
\text { size }\end{array}$ & $\begin{array}{l}\text { Gp20 } \\
\text { product }\end{array}$ & $\begin{array}{l}\text { Host range } \\
\text { WH7803 }\end{array}$ & WH8103 & RS9906 & RS9911 & SS120 \\
\hline S-RSM3 & 7.9 & 150 & $154 \pm 6$ & $\checkmark$ & $\bullet$ & {$[\bullet]$} & 0 & O & 0 \\
\hline S-RSM4 & 27.4 & 50 & $154 \pm 6$ & $\checkmark$ & $\bullet$ & {$[\bullet]$} & O & O & 0 \\
\hline S-RSM5 & 27.12 & 150 & $170 \pm 5$ & $\checkmark$ & O & {$[\bullet]$} & O & O & 0 \\
\hline S-RSM6 & 19.4 & 50 & $155 \pm 10$ & $\checkmark$ & $\bullet$ & {$[\bullet]$} & O & O & 0 \\
\hline S-RSM7 & 27.4 & 10 & & $\checkmark$ & O & {$[\bullet]$} & O & O & 0 \\
\hline S-RSM8 & 11.5 & 0 & $161 \pm 8$ & $\checkmark$ & $\bullet$ & $\bullet$ & $\bullet$ & O & 0 \\
\hline S-RSM9 & 19.4 & 0 & $155 \pm 4$ & $\checkmark$ & $\bullet$ & {$[\bullet]$} & o & 0 & 0 \\
\hline S-RSM10 & 19.4 & 125 & & $\checkmark$ & {$[\bullet]$} & 0 & 0 & 0 & 0 \\
\hline S-RSM11 & 27.4 & 10 & $153 \pm 3$ & $\checkmark$ & $\bullet$ & {$[\bullet]$} & 0 & o & $\bullet$ \\
\hline S-RSM12 & 27.12 & 30 & & $\checkmark$ & {$[\bullet]$} & 0 & O & ○ & O \\
\hline S-RSM13 & 19.4 & 60 & $161 \pm 7$ & $\checkmark$ & $\bullet$ & {$[\bullet]$} & O & O & O \\
\hline S-RSM14 & 4.10 & 80 & $157 \pm 9$ & $\checkmark$ & $\bullet$ & {$[\bullet]$} & $\circ$ & ○ & 0 \\
\hline S-RSM15 & 19.4 & 10 & $156 \pm 3$ & $\checkmark$ & {$[\bullet]$} & $\bullet$ & 0 & O & O \\
\hline S-RSM16 & 11.5 & 125 & $151 \pm 6$ & $\checkmark$ & $\bullet$ & {$[\bullet]$} & 0 & 0 & 0 \\
\hline S-RSM17 & 19.4 & 80 & $160 \pm 3$ & $\checkmark$ & {$[\bullet]$} & 0 & 0 & O & O \\
\hline S-RSM18 & 11.5 & 50 & $157 \pm 3$ & $\checkmark$ & $\bullet$ & {$[\bullet]$} & 0 & O & 0 \\
\hline S-RSM19 & 27.4 & 80 & $165 \pm 6$ & $\checkmark$ & $\bullet$ & {$[\bullet]$} & O & O & o \\
\hline S-RSM20 & 4.10 & 50 & $162 \pm 8$ & $\checkmark$ & $\bullet$ & {$[\bullet]$} & O & O & 0 \\
\hline S-RSM21 & 18.10 & 100 & & $\checkmark$ & $\bullet$ & {$[\bullet]$} & O & O & 0 \\
\hline S-RSM22 & 7.9 & 125 & $152 \pm 1$ & $\checkmark$ & $\bullet$ & {$[\bullet]$} & 0 & o & 0 \\
\hline S-RSM23 & 18.10 & 125 & & $\checkmark$ & $\bullet$ & {$[\bullet]$} & $\bullet$ & 0 & 0 \\
\hline S-RSM24 & 11.5 & 100 & $154 \pm 2$ & $\checkmark$ & $\bullet$ & {$[\bullet]$} & o & O & 0 \\
\hline S-RSM25 & 27.4 & 60 & $155 \pm 3$ & $\checkmark$ & $\bullet$ & {$[\bullet]$} & O & ○ & O \\
\hline S-RSM26 & 27.4 & 50 & $154 \pm 9$ & $\checkmark$ & $\bullet$ & {$[\bullet]$} & O & ○ & O \\
\hline S-RSM27 & 18.10 & 125 & & $\checkmark$ & $\bullet$ & {$[\bullet]$} & 0 & O & 0 \\
\hline S-RSM28 & 11.5 & 50 & $154 \pm 10$ & $\checkmark$ & $\bullet$ & {$[\bullet]$} & ○ & O & O \\
\hline S-RSM29 & 19.4 & 30 & $152 \pm 6$ & $\checkmark$ & $\bullet$ & {$[\bullet]$} & 0 & O & O \\
\hline S-RSM30 & 4.10 & 50 & $163 \pm 3$ & $\checkmark$ & $\bullet$ & {$[\bullet]$} & 0 & 0 & 0 \\
\hline S-RSM31 & 18.1 & 125 & & $\checkmark$ & $\bullet$ & {$[\bullet]$} & 0 & O & 0 \\
\hline S-RSM32 & 19.4 & 60 & $152 \pm 3$ & $\checkmark$ & $\bullet$ & {$[\bullet]$} & ○ & ○ & $\bullet$ \\
\hline S-RSM33 & 18.10 & 125 & & $\checkmark$ & $\bullet$ & {$[\bullet]$} & $\bullet$ & o & O \\
\hline S-RSM34 & 27.4 & 60 & $151 \pm 4$ & $\checkmark$ & $\bullet$ & {$[\bullet]$} & O & O & 0 \\
\hline S-RSM35 & 27.4 & 80 & $152 \pm 3$ & $\checkmark$ & $\bullet$ & {$[\bullet]$} & 0 & O & 0 \\
\hline S-RSM36 & 18.10 & 125 & $156 \pm 4$ & $\checkmark$ & • & {$[\bullet]$} & 0 & 0 & 0 \\
\hline S-RSM37 & 22.11 & 150 & $175 \pm 6$ & $\checkmark$ & $\bullet$ & {$[\bullet]$} & $\bullet$ & O & O \\
\hline S-RSM38 & 27.4 & 60 & & $\checkmark$ & $\bullet$ & {$[\bullet]$} & ○ & ○ & ○ \\
\hline S-RSM39 & 27.4 & 30 & $192 \pm 5$ & $\checkmark$ & $\bullet$ & {$[\bullet]$} & o & ○ & O \\
\hline S-RSM40 & 19.4 & 60 & $197 \pm 6$ & $\checkmark$ & {$[\bullet]$} & $\bullet$ & O & O & O \\
\hline S-RSM41 & 19.4 & 60 & $194 \pm 4$ & $\checkmark$ & {$[\bullet]$} & $\bullet$ & 0 & 0 & O \\
\hline S-RSM42 & 19.4 & 80 & $162 \pm 10$ & $\checkmark$ & {$[\bullet]$} & $\bullet$ & o & O & $\bullet$ \\
\hline S-RSM43 & 27.4 & 50 & $189 \pm 7$ & $\checkmark$ & {$[\bullet]$} & o & 0 & 0 & o \\
\hline S-RSM44 & 27.4 & 30 & & $\checkmark$ & {$[\bullet]$} & $\bullet$ & 0 & 0 & $\bullet$ \\
\hline S-RSM45 & 27.4 & 80 & $154 \pm 3$ & $\checkmark$ & {$[\bullet]$} & • & O & O & $\bullet$ \\
\hline S-RSM46 & 27.4 & 80 & $160 \pm 9$ & $\checkmark$ & {$[\bullet]$} & O & o & O & O \\
\hline S-RSM47 & 19.4 & 30 & & $\checkmark$ & {$[\bullet]$} & $\bullet$ & 0 & O & 0 \\
\hline S-RSM48 & 11.5 & 50 & $151 \pm 2$ & $\checkmark$ & {$[\bullet]$} & $\bullet$ & 0 & O & 0 \\
\hline S-RSM49 & 11.5 & 50 & $158 \pm 5$ & $\checkmark$ & {$[\bullet]$} & • & $\bullet$ & o & 0 \\
\hline S-RSM50 & 27.4 & 80 & $159 \pm 3$ & $\checkmark$ & {$[\bullet]$} & o & o & 0 & 0 \\
\hline S-RSM51 & 11.5 & 80 & $153 \pm 3$ & $\checkmark$ & {$[\bullet]$} & $\bullet$ & $\circ$ & O & 0 \\
\hline S-RSM52 & 11.5 & 80 & $192 \pm 1$ & $\checkmark$ & {$[\bullet]$} & • & 0 & 0 & 0 \\
\hline S-RSM53 & 11.5 & 80 & $167 \pm 6$ & & {$[\bullet]$} & $\bullet$ & 0 & 0 & 0 \\
\hline S-RSM54 & 11.5 & 80 & & $\checkmark$ & {$[\bullet]$} & O & o & O & $\bullet$ \\
\hline S-RSM55 & 11.5 & 80 & $185 \pm 13$ & $\checkmark$ & {$[\bullet]$} & $\bullet$ & 0 & 0 & O \\
\hline S-RSM56 & 11.5 & 80 & $153 \pm 6$ & $\checkmark$ & {$[\bullet]$} & • & O & ○ & $\bullet$ \\
\hline S-RSM57 & 11.5 & 100 & & $\checkmark$ & {$[\bullet]$} & o & 0 & 0 & $\bullet$ \\
\hline S-RSM58 & 11.5 & 150 & $152 \pm 8$ & $\checkmark$ & {$[\bullet]$} & $\bullet$ & 0 & 0 & o \\
\hline S-RSM59 & 14.6 & 60 & $150 \pm 4$ & $\checkmark$ & {$[\bullet]$} & $\bullet$ & O & O & 0 \\
\hline
\end{tabular}


Table 1. Continued.

\begin{tabular}{|c|c|c|c|c|c|c|c|c|c|}
\hline Phage & $\begin{array}{l}\text { Date of } \\
\text { isolation }\end{array}$ & Depth & $\begin{array}{l}\text { Genome } \\
\text { size }\end{array}$ & $\begin{array}{l}\text { Gp20 } \\
\text { product }\end{array}$ & $\begin{array}{l}\text { Host range } \\
\text { WH7803 }\end{array}$ & WH8103 & RS9906 & RS9911 & SS120 \\
\hline S-RSM60 & 18.7 & 30 & & $\sqrt{ }$ & {$[\bullet]$} & $\bullet$ & o & 0 & o \\
\hline S-RSM61 & 18.7 & 30 & & $\sqrt{ }$ & {$[\bullet]$} & $\bullet$ & ○ & O & o \\
\hline S-RSM62 & 18.7 & 150 & $179 \pm 6$ & $\sqrt{ }$ & {$[\bullet]$} & $\bullet$ & O & O & 0 \\
\hline S-RSM63 & 23.8 & 80 & & $\checkmark$ & {$[\bullet]$} & O & 0 & O & O \\
\hline S-RSM64 & 7.9 & 30 & $156 \pm 6$ & $\checkmark$ & {$[\bullet]$} & $\bullet$ & 0 & O & 0 \\
\hline S-RSM65 & 7.9 & 80 & $167 \pm 3$ & & {$[\bullet]$} & $\bullet$ & 0 & 0 & 0 \\
\hline S-RSM66 & 18.10 & 125 & $153 \pm 6$ & $\checkmark$ & {$[\bullet]$} & - & $\bullet$ & 0 & 0 \\
\hline S-RSM67 & 18.10 & 125 & & $\checkmark$ & {$[\bullet]$} & $\bullet$ & $\bullet$ & $\bullet$ & 0 \\
\hline S-RSM68 & 18.7 & 150 & & $\checkmark$ & {$[\bullet]$} & o & 0 & o & 0 \\
\hline S-RSM69 & 18.7 & 125 & $189 \pm 13$ & $\checkmark$ & {$[\bullet]$} & $\bullet$ & o & O & O \\
\hline S-RSM70 & 23.8 & 10 & $153 \pm 3$ & & {$[\bullet]$} & $\bullet$ & o & O & O \\
\hline S-RSM71 & 18.10 & 30 & & $\checkmark$ & {$[\bullet]$} & O & o & O & ○ \\
\hline S-RSM72 & 18.7 & 10 & $197 \pm 11$ & $\sqrt{ }$ & {$[\bullet]$} & O & o & O & O \\
\hline S-RSM73 & 27.12 & 30 & $179 \pm 4$ & $\checkmark$ & {$[\bullet]$} & 0 & o & 0 & 0 \\
\hline S-RSM74 & 11.5 & 80 & $156 \pm 10$ & $\checkmark$ & {$[\bullet]$} & $\bullet$ & ○ & O & O \\
\hline S-RSM75 & 27.4 & 30 & $151 \pm 14$ & $\checkmark$ & {$[\bullet]$} & $\bullet$ & o & 0 & 0 \\
\hline S-RSM76 & 19.4 & 125 & $183 \pm 6$ & $\checkmark$ & {$[\bullet]$} & $\bullet$ & o & O & O \\
\hline S-RSM77 & 11.5 & 50 & $154 \pm 4.4$ & $\checkmark$ & {$[\bullet]$} & $\bullet$ & 0 & O & 0 \\
\hline S-RSM78 & 18.7 & 150 & $180 \pm 6$ & & {$[\bullet]$} & O & ○ & O & O \\
\hline S-RSM79 & 18.10 & 80 & & $\checkmark$ & {$[\bullet]$} & 0 & 0 & O & O \\
\hline S-RSM80 & 11.5 & 80 & $185 \pm 16$ & $\checkmark$ & {$[\bullet]$} & $\bullet$ & 0 & 0 & O \\
\hline S-RSM81 & 19.4 & 80 & $151 \pm 3$ & $\checkmark$ & {$[\bullet]$} & $\bullet$ & 0 & 0 & 0 \\
\hline S-RSM82 & 11.5 & 80 & $195 \pm 13$ & $\checkmark$ & {$[\bullet]$} & $\bullet$ & o & 0 & 0 \\
\hline S-RSM83 & 19.4 & 30 & & & {$[\bullet]$} & O & o & O & O \\
\hline S-RSM84 & 19.4 & 125 & $186 \pm 12$ & $\checkmark$ & {$[\bullet]$} & $\bullet$ & o & O & ○ \\
\hline S-RSM85 & 19.4 & 125 & $189 \pm 8$ & $\checkmark$ & {$[\bullet]$} & O & o & O & 0 \\
\hline S-RSM86 & 19.4 & 125 & $201 \pm 4$ & $\checkmark$ & {$[\bullet]$} & 0 & o & O & 0 \\
\hline S-RSM87 & 19.4 & 125 & $204 \pm 7$ & $\checkmark$ & {$[\bullet]$} & O & ○ & O & O \\
\hline S-RSM88 & 19.4 & 125 & $155 \pm 3$ & $\checkmark$ & - & O & {$[\bullet]$} & O & O \\
\hline S-RS89* & 19.4 & 125 & $198 \pm 16$ & $\checkmark$ & {$[\bullet]$} & o & 0 & 0 & O \\
\hline
\end{tabular}

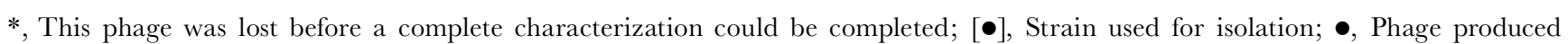
plaques/clearing of wells; $\bigcirc$, No plaques produced/no clearing of wells; $\checkmark$, A gp20 product was obtained using the primers CPS-1 and CPS-2.

placed in a lysis buffer of $0.1 \mathrm{M}$ EDTA, 1\% SDS, $0.1 \mathrm{M}$ Tris- $\mathrm{HCl} \mathrm{pH} \mathrm{9.0,} 0.5 \mathrm{mg} \mathrm{ml}^{-1}$ proteinase $\mathrm{K}$ and incubated at $55^{\circ} \mathrm{C}$ overnight. The plugs were washed twice with $200 \mathrm{mM}$ TE, and stored at $4^{\circ} \mathrm{C}$ until ready for use. Samples were run in a 1\% PFGE-grade agarose gel (BioRad) with lambda PFGE ladders (Sigma) run in the outside lanes. Gels were run initially in a CHEF Mapper (Bio-Rad) in $0.5 \times \mathrm{TBE}$ at $14^{\circ} \mathrm{C}, 6 \mathrm{~V} \mathrm{~cm}^{-1}$ with an initial switch time of $6.75 \mathrm{~s}$ and a final switch time of $21.79 \mathrm{~s}$ for $20 \mathrm{~h}$. After it became apparent that no isolate had a genome size of less than $100 \mathrm{~kb}$ the switch times were changed to an initial switch time of $12.55 \mathrm{~s}$ and a final switch time of $17.33 \mathrm{~s}$, all other conditions were kept constant. Gels were stained with ethidium bromide $\left(0.5 \mu \mathrm{gl}^{-1}\right)$ for $1 \mathrm{~h}$ and then washed in deionized $\mathrm{H}_{2} \mathrm{O}$ for 20 min. Gel images were digitally captured using a Syngene gel documentation system (Cambridge, UK). A genome size for each phage was estimated by taking the average genome size from a minimum of three replicates.

\section{RESULTS}

\section{Cyanophage abundance}

It was only possible to enumerate phages from April 1999 to December 1999 and this period will be referred to as the annual cycle, although it does not cover a full 12 months. Phage abundance was determined using four Synechococcus strains, but phage numbers were found to be highest when Synechococcus sp. WH7803 was used as a host. Cyanophages were found at all depths throughout the annual cycle, but were not found using all strains of Synechococcus as hosts. Titres of cyanophages ranged from undetectable to $6.08 \times 10^{3} \mathrm{pfu} \mathrm{ml}^{-1}$ (Figure $1 \mathrm{~A}-\mathrm{K}$ ).

For the months of April, May and June the abundance of cyanophages was low with often no phages detected at some depths when strains WH8103, RS9906 and RS9911 were used as hosts (Figure $1 \mathrm{~A}-\mathrm{D}$ ). In July an increase in phage number was found at $10 \mathrm{~m}$ and a maximum in the number of phages infecting Synechococcus sp. WH7803 was observed at a depth of $30 \mathrm{~m}$ (Figure 1E). It was at this depth that the highest cyanophage abundance $\left(6.08 \times 10^{3} \mathrm{pfuml}^{-1}\right)$ of the entire annual cycle was observed in September (Figure $1 \mathrm{G}$ ). This pattern was not observed when RS9906, RS9911 and WH8103 were used to estimate phage abundance (Figure $1 \mathrm{E}-\mathrm{G}$ ). The high abundance of phage at $30 \mathrm{~m}$ continued into August and September, but by early October abundance had started to decline and by late October the decline was complete (Figure $1 \mathrm{~F}-\mathrm{I}$ ). During August, September and early October there was a small increase in phage numbers, 


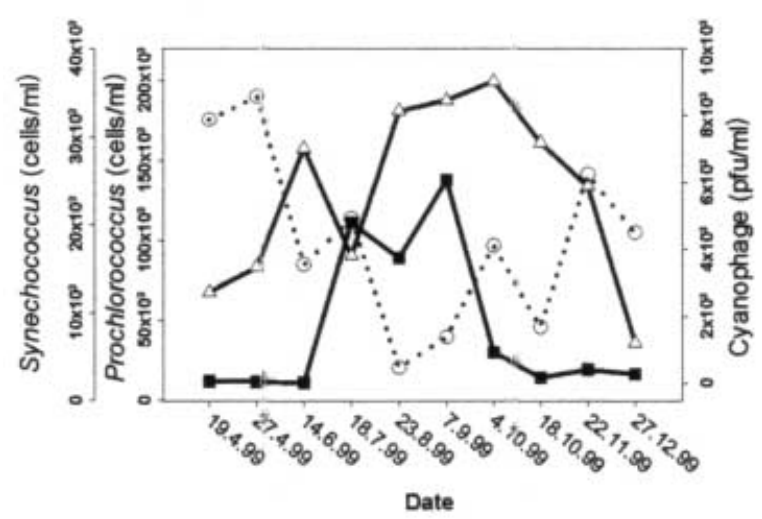

Figure 2. Synechococcus, Prochlorococcus and cyanophage abundance during the annual cycle in the Gulf of Aqaba at a depth of $30 \mathrm{~m}$. Cyanophage numbers are representative of those obtained using Synechococcus sp. WH7803 as a host (ש). Synechococcus $(\mathrm{O})$ and Prochlorococcus $(\triangle)$ numbers were from flow cytometry counts from previously published data (Fuller et al., 2005).

most notably at a depth of $150 \mathrm{~m}$ in August and September (Figure $1 \mathrm{G}, \mathrm{H}$ ).

Using Synechococcus strains WH8103, RS9906 and RS9911 as hosts, far lower phage titres were obtained (Figure 1A-K). Phages capable of infecting these strains were detected throughout the year in very low abundances, however, the highest numbers of phage found were in late October, November and December. Host strains RS9906 and RS9911 revealed a similar profile of phage abundance, with maxima at $50 \mathrm{~m}$ in November and December (Figure 1J,K). When WH8103 was used as a host the maximum in phage abundance was found at a depth of $100 \mathrm{~m}$ (Figure 1J). However, this peak of $5 \times 10^{2} \mathrm{pfu} \mathrm{ml}^{-1}$ was an order of magnitude lower than the titre of phages found to infect WH7803 at a depth of $30 \mathrm{~m}$ in July.

The abundances of cyanophages and their potential Synechococcus hosts at $30 \mathrm{~m}$ do not covary (Figure 2). When Synechococcus numbers are at their highest during the months of November, December and April the numbers of cyanophages were at their lowest. Using Pearson's correlation it was found there was no significant correlation between the abundance of cyanophages and Prochlorococcus $(-0.51)$ or Synechococcus $(0.33)$ for the entire annual cycle. However, it should be noted that the maxima in cyanophage abundance during July, August and September coincide with maximal Prochlorococcus abundance (Figure 2).

\section{Host range}

The host used for cyanophage enumeration was found to be important in estimating cyanophage numbers. Of all the plaques counted, 88\% were observed on WH7803 plates. With $6 \%, 4 \%$ and $2 \%$ of total plaques found when using RS9906, WH8103 and RS9911 as hosts respectively. A total of 87 cyanophages was isolated using different hosts and were characterized in terms of their ability to infect the other strains of Synechococcus used in estimating phage abundance. It was found that $\sim 98 \%$ of phage were capable of infecting Synechococcus WH7803 and $75 \%$ capable of infecting WH8103 (Table 1). Far fewer phages could infect the other two strains used, with $~ 9 \%$

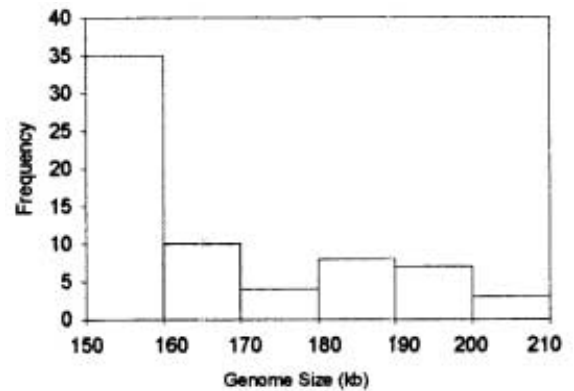

Figure 3. Histogram of the genome size of 67 cyanophages isolated from the Red Sea.

and $\sim 1 \%$ capable of infecting RS9906 and RS9911 respectively (Table 1). The ability of phage isolates to infect multiple hosts was found to be widespread with $\sim 77 \%$ of phage infecting at least two strains. However, only phage S-RSM67 was capable of infecting all four strains (Table 1). The host range of isolates was correlated with the strain used for isolation. Sixty-five per cent of the phages isolated on WH7803 could infect WH8103, whereas of the phages isolated on WH8103 91\% could also infect WH7803 (Table 1).

The coincidence of the maxima in cyanophage abundance during July, August and September with maximal Prochlorococcus abundance (Figure 2) suggests that cyanophages being titred on Synechococcus may also be capable of infecting Prochlorococcus strains. To test this hypothesis the 87 Red Sea cyanophage isolates, S-PM2, and S-WHMl were screened for their ability to infect Prochlorococcus strain SS120. It was found for the phages S-WHM1，S-RS11，S-RS33，S-RS42，S-RS44，S-RS45, S-RS54, S-RS56 and S-RS57 could cause the clearing of cultures of Prochlorococcus (Table 1) and therefore might be assumed to be infective. However, it was not possible to determine whether this was true infection and lysis or some phenomenon like lysis from without, where a number of phage absorb to and lyse a cell without the production of progeny phage (Delbruck, 1940). In the case of phage S-WHMl, the titre of phage added to the Prochlorococcus was $2.64 \times 10^{6} \mathrm{pfu} \mathrm{ml}^{-1}$ using Synechococcus sp. WH7803 as a host, but after apparent lysis the supernatant had a phage titre of only $1.67 \times 10^{6}$ pfu ml ${ }^{-1}$.

\section{Cyanophage classification}

A total of 87 cyanophages were isolated from samples obtained at different times and depths through the annual cycle. The phage isolates were screened by PCR using the cyanomyovirus-specific primers CPS-1 and CPS-2 (Fuller et al., 1998). When a correctly sized 165 bp PGR product was obtained the phage in question was classified as a cyanomyovirus. A negative result was taken to be inconclusive, as the primers are known not to amplify g20 from all cyanomyoviruses (Fuller et al., 1998).

It was possible to identify $82 / 87$ isolates as being members of the Myoviridae family by using this PCR approach (Table 1). The remaining unidentified isolates RS53, RS65, RS70, RS78 and RS83 were examined by TEM to try to determine which family of viruses they belong to. In each case the phages were found to have 
tails of variable lengths, suggesting that the tail is contractile, and therefore that the phage is a member of the Myoviridae family. Thus all the isolates were identified as cyanomyoviruses.

\section{Genome size}

It was possible to determine the genome size of 67 of the 87 cyanophage by the use of PFGE. The cyanophage S-PM2 was used as control as it has previously been estimated to have a genome size of $194 \mathrm{~kb}$ by PFGE (Hambly et al., 2001) and is now known to have an actual genome size of $196.28 \mathrm{~kb}$ (Mann et al., 2005). In this study S-PM2 was estimated to have the genome size of $197 \pm 4 \mathrm{~kb}$ indicating that PFGE was yielding an accurate estimation of genome size. The smallest estimated genome size in this study was that of cyanophage S-RSM59, which had a genome size of $150 \pm 4 \mathrm{~kb}$ and the largest had genomes in excess of $200 \mathrm{~kb}$ (Figure 3).

\section{DISCUSSION}

Previous studies have examined cyanophage numbers over an annual cycle, and found that cyanophage numbers are not constant over the period. In a study of cyanophage numbers from March 2001 to October 2002 in Mt Hope Bay, Rhode Island, it was found there was a seasonality to cyanophage numbers (Marston \& Sallee, 2003). This seasonal variation was partly attributed to the variation in Synechococcus abundance, as previous studies have found that cyanophage abundance is closely related to Synechococcus abundance (Waterbury \& Rippka, 1989; Lu et al., 2001). In a study in the Gulf of Mexico it was found that phage abundance was closely linked to Synechococcus abundance, with a threshold value of $10^{3} \mathrm{ml}^{-1}$ Synechococcus cells, above which phage numbers increased from $10^{2}$ to $10^{5} \mathrm{ml}^{-1}$ (Suttle \& Chan, 1994). In this study where cyanophage numbers increased 100-fold from June to July at a depth of $30 \mathrm{~m}$ (Figure 2), the total number of Synechococcus has been found to decrease, but it did stay above the threshold value of $10^{3}$ cells $\mathrm{ml}^{-1}$ (Fuller et al., 2005). This decrease in Synechococcus numbers and increase in cyanophage is in contradiction to other reports of cyanobacteria/phage relationships (Waterbury \& Valois, 1993; Sullivan et al., 2003). It is important to realize that the decrease in Synechococcocus numbers is only representative of the total Synechococcus population as determined by flow cytometer counts. These counts are unable to detect any subtle changes in Synechococcus populations. Therefore, the increase in a population of hosts susceptible to infection could be masked by the net decrease in the abundance of all other Synechococcus populations.

There is also the possibility that there is a host other than Synechococcus that the cyanophages can infect. It was observed that although Synechococcus numbers decreased during the cyanophage maxima in the period from July to September, the number of Procholorococcus was still very high (Figure 2). It is possible that cyanophages capable of infecting both Synechococcus and Procholorococcus were being detected. It is known that some cyanophages can infect both Synechococcus and Procholorococcus (Sullivan et al., 2003). A parallel study using the same water samples revealed that the genetic diversity of Prochlorococcus changed over the same annual cycle (Fuller et al., 2005). Using 16S rRNA dot blot hybridization high-light (HL)Prochlorococcus genotypes were found to dominate from April to December and low-light (LL)-Prochlorococcus were found from July to early October. By midOctober LL-Prochlorococcus genotypes were not detected. This observation is important as Sullivan et al. (2003) found cyanophages that were able to infect both Synechococcus and LL-Prochlorococcus, but not Synechococcus and HL-Prochlorococcus (Sullivan et al., 2003). The increase in LL-Prochlorococcus in the Red Sea during the month of August also coincided with an increase in the abundance of cyanophages at a depth of $30 \mathrm{~m}$ (Figure 2); the decrease by mid October also coincided with the decrease in phage numbers at $30 \mathrm{~m}$. The hypothesis that cyanophages may infect Prochlorococcus was tested by attempting to infect Prochlorococcus strain SS120 using the 87 Red Sea cyanophage isolates, together with phages S-PM2 and S-WHM1 as negative and positive controls respectively. It was found that several phage isolates, including S-WHM1, could cause the clearing of cultures of Procholorococcus. However, it was not possible to determine whether this was directly caused by the addition of phage leading to infection and subsequent lysis. In the case of phage $\mathrm{S}-\mathrm{WHMl}$ there was no increase in phage titre after lysis of the Prochlorococcus culture, as measured using Synechococcus sp. WH7803 as a host. This could suggest that S-WHM1 does not infect Prochlorococcus sp. SS120, which would be in conflict with a previous report (Sullivan et al., 2003). Alternatively, it could be that after infection of Prochlorococcus the progeny phage lacked the ability to efficiently infect Synechococcus as a result of altered DNA modification or the production of phage virions with alternative adhesins. However, it was not possible to distinguish between these possibilities without the ability to grow Prochlorococcus on agar plates and detect individual plaques.

\section{Host range}

It was apparent that the number of cyanophages that were detected is dependent on the host strain of Synechococcus used for enumeration (Figure 1A-K). A similar observation was made previously by Waterbury \& Valois (1993) who found that cyanophage numbers varied from $3.5 \times 10^{1}$ when using Synechococcus sp. WH8108 as a host compared to $7 \times 10^{3}$ with host strain Synechococcus sp. WH8017. Lu et al. (2001), also found that on average phage numbers were 324 times higher using Synechococcus sp. WH7803 as a host compared to Synechococcus sp. WH8101.

As Synechococcus sp. WH7803 gave the highest estimation of phage abundance (Figure 1) it was unsurprising that the majority of phages were capable of infecting the Synechococcus WH7803 in the host range studies (Table 1). However, $\sim 75 \%$ of phage isolates were found to infect WH8103, yet the abundance of phages estimated with WH8103 used were significantly lower than when WH7803 was used as host for phage enumeration.

Host range has previously been linked to phage morphology. For example, phages that infect HL-Prochlorococcus have been shown to be exclusively podoviruses and host specific, while myoviruses could infect multiple strains of Synechococcus or Prochlorococcus and some infect both genera (Sullivan et al., 2003). All phages isolated in this study are thought to be 
myoviruses, yet it was found that some phages such as SRSM12 could infect only one strain while others such as SRSM67 could infect all fours strains of Synechococcus tested (Table 1).

The host range of cyanophages have previously been studied in some detail (Suttle \& Chan, 1993, 1994; Waterbury \& Valois, 1993; Wilson et al., 1993; Lu et al., 2001). Marine unicellular cyanobacteria possessing phycoerythrin as their primary accessory light-harvesting pigment were originally classified as marine cluster A (MC-A) Synechococcus and distinguished from marine cluster B (MC-B), members of which have phycocyanin as their major light-harvesting pigment (Waterbury \& Rippka, 1989). The recent division of the genus Synechococcus into five clusters (Herdman et al., 2001) makes the comparison with previous studies difficult. Marine Synechococcus now comprise sub-clusters 5.1 and 5.2. The new sub-cluster 5.1 is representative of the majority of what was previously MC-A and sub-cluster 5.2 represents the majority of what was MC-B. For the purpose of comparison with previous studies, strains in this study will still be referred to as MC-A or MC-B. The MC-A strains can be divided into ten clades by the phylogenetic analysis of 16S rDNA (Fuller et al., 2003). All strains used in this study are MC-A strains, with Synechococcus sp. WH7803 a representative of clade V, Synechococcus sp. WH8103 a representative of clade III, Synechococcus sp. RS9906 a representative of clade VIII and Synechococcus sp. RS9911 a representative of clade II. These four clades are monophyletic and have all evolved from a common ancestral host. Clades VIII and III show greater evolutionary distance from the common ancestor than clade II and clade V (Fuller et al., 2003). It therefore might be expected that a phage is more likely to infect both a clade II and clade V Synechococcus than a clade II and clade VIII Synechococcus.

The host range of cyanophages, in common with phage morphology, has previously been shown to be linked to the strain of Synechococcus used for isolation. Suttle \& Chan (1994) isolated phages infecting a MC-A Synechococcus and a green-coloured strain (possibly MC-B) of Synechococcus. They found that there was greater cross infection between MC-A strains than between MC-A strains and the green strain of Synechococcus. The results of this study (Table 1) indicate that there is greater cross infection between WH7803 (clade V) and WH8103 (clade II) than between RS9906 (clade VIII) and WH7803 (clade V) or RS9906 (clade VIII) and WH8103 (clade III). These results were similar to that of Suttle \& Chan (1993) as both WH7803 and WH8103 are orange/red-coloured where as RS9906 is a green-coloured strain of Synechococcus. The comparison with previous studies is made difficult as there is no genetic data available for all strains used in previous studies, so it cannot be determined what genotype a strain may be. New observations contrary to previous studies (Suttle \& Chan, 1993) were found in this study, with only two of the isolated phages infecting the clade II strain Synechococcus sp. RS9911 (also a red/orange-coloured strain), in contrast to the eight that infected the green RS9906 (clade VIII strain). The results therefore indicate that more phages do not cross infect Synechococcus from clade II and VIII as might be expected but show a greater cross infectivity between clade V (WH7803) and clade III (WH8103). As current Synechococcus phylogeny is based on 16S rDNA, it is unlikely to be useful in the prediction of which strains of Synechococcus a phage can infect, as there will be no direct selective pressure on the 16S rDNA of Synechococcus by a phage. Whereas the phylogeny of receptors on the host are much more likely to predict what phage a host can infect. However, until the receptors on the host are identified and the tail fibres in phages are found, the ability of phages to infect multiple strains of Synechococcus is unlikely to be fully explained.

The ability of the cyanophages in this study to infect multiple hosts, also fits well with the theory of Wommack \& Colwell (Wommack \& Colwell, 2000), that phage from oligotrophic environments have broader host ranges. The thinking behind this hypothesis is that phage from oligotrophic water will encounter hosts less often, and will therefore have an advantage if they can infect more than one host, as they are more likely to encounter a host they can infect.

It has been proposed that the differences in the host ranges of cyanophages will be influenced by contact rates between host cells and phage. When contact rates are high, there will be a strong selection for resistant cells; when contact rates are lower there will be less selection pressure for resistant hosts (Waterbury \& Valois, 1993; Suttle \& Chan, 1994). In the Red Sea, clade II strains of Synechococcus have been found to dominate throughout an annual cycle and RS9911 is a representative of this clade (Fuller et al., 2003). It could be that RS9911 and its relatives are resistant to most of the co-occurring phages and this allows their dominance. However, no studies have yet systematically looked at patterns of phage resistance within a clade.

This study has shown that in an oligotrophic body of water representative of an open ocean system there is seasonal variation in cyanophage numbers as was previously found in coastal studies (Suttle \& Chan, 1993; Waterbury \& Valois, 1993; Marston \& Sallee, 2003). However, it has also revealed the difficulty with currently available techniques of identifying the true impact of phage infection on natural marine cyanobacterial communities. It is impossible to assess the total infectivity of phages present in a given environment with respect to a complex community of potential hosts and the potential phage:host interactions are complicated by abiotic variables such as nutrient availability, light intensity etc. A further convolution is the impact of grazers on this system. Future studies on the nature of the phage receptors on the surface of Synechococcus and Prochlorococcus cells and phage adhesins may provide us with both an understanding of these phage:host interactions and the molecular tools with which to quantify them in situ.

This work was part funded by the Natural Environment Research Council grants awarded to N.H. Mann. We gratefully thank N. Fuller for his collection of water samples from the Red Sea that were used in this study.

\section{REFERENCES}

Delbruck, M., 1940. The growth of bacteriophage and lysis of the host. Journal of General Physiology, 23, 643-660. 
Frederickson, C.M., Short, S.M. \& Suttle, C.A., 2003. The physical environment affects cyanophage communities in British Columbia inlets. Microbial Ecology, 46, 348-357.

Fuller, N.J., Marie, D., Partensky, F., Vaulot, D., Post, A.F. \& Scanlan, D.J., 2003. Clade-specific 16S ribosomal DNA oligonucleotides reveal the predominance of a single marine Synechococcus clade throughout a stratified water column in the Red Sea. Applied and Environmental Microbiology, 69, 2430-2443.

Fuller, N.J., West, N.J., Marie, D., Yallop, M., Rivlin, T., Post, A.F. \& Scanlan, D.J., 2005. Dynamics of community structure and phosphate status of picocyanobacterial populations in the Gulf of Aqaba, Red Sea. Limnology and Oceanography, 50, 363375.

Fuller, N.J., Wilson, W.H., Joint, I.R. \& Mann, N.H., 1998. Occurrence of a sequence in marine cyanophages similar to that of T4 g20 and its application to PCR-based detection and quantification techniques. Applied and Environmental Microbiology, 64, 2051-2060.

Hambly, E., Tétart, F., Desplats, C., Wilson, W.H., Krisch, H.M. \& Mann, N.H., 2001. A conserved genetic module that encodes the major virion components in both the coliphage T4 and the marine cyanophage S-PM2. Proceedings of the National Academy of Sciences of the United States of America, 98, $11411-11416$.

Herdman, M., Castenholz, R.W., Iteman, I., Waterbury, J.B. \& Rippka, R., 2001. The Archaea and the deeply branching and phototrophic bacteria. In Bergey's manual of systematic biology (ed. D.R. Boone et al.), pp. 493-514. Springer Publishers.

Klinker, J., Reiss, Z., Kropach, C., Levanon, I., Harpaz, H. \& Halicz, E., 1976. Observations on the circulation pattern in the Gulf of Elat (Aqaba), Red Sea. Israel Journal of Earth Sciences, 25, 85-103.

Lu, J., Chen, F. \& Hodson, R.E., 2001. Distribution, isolation, host specificity, and diversity of cyanophages infecting marine Synechococcus spp. in river estuaries. Applied and Environmental Microbiology, 67, 3285-3290.

Mann, N.H., Glokie, M.R., Millard, A., Cook, A., Wilson, W.H., Wheatley, P.J., Letarov, A. \& Krisch, H.M., 2005. The genome of S-PM2, a 'photosynthetic' T4-type bacteriophage that infects marine Synechococcus strains. Fournal of Bacteriology, 187, 3188-3200.

Mann, N.H., Cook, A., Millard, A., Bailey, S. \& Clokie, M., 2003. Marine ecosystems: bacterial photosynthesis genes in a virus. Nature, London, 424, 741-741.

Marston, M.F. \& Sallee, J.L., 2003. Genetic diversity and temporal variation in the cyanophage community infecting marine Synechococcus species in Rhode Island's coastal waters. Applied and Environmental Microbiology, 69, 4639-4647.
Mühling M. et al., 2005. Genetic diversity of marine Synechococcus and co-occurring cyanophage communities: evidence for viral control of phytoplankton. Environmental Microbiology, 7, 499508.

Partensky, F., Hess, W.R. \& Vaulot, D., 1999. Prochlorococcus, a marine photosynthetic prokaryote of global significance. Microbiology and Molecular Biology Reviews, 63, 106-127.

Reiss, Z. \& Hottinger, L., 1984. The Gulf of Aqaba (Elat): ecological micropaleontology (Ecological Studies, Vol. 50). New York: SpringerVerlag.

Sullivan, M.B., Waterbury, J.B. \& Chisholm, S.W., 2003. Cyanophages infecting the oceanic cyanobacterium Prochlorococcus. Nature, London, 424, 1047-1051.

Suttle, C.A., 2000. Cyanophages and their role in the ecology of cyanobacteria. In The ecology of cyanobacteria: their diversity in time and space (ed. B.A. Whitton and M. Potts). Boston: Kluwer Academic Publishers.

Suttle, C.A. \& Chan, A.M., 1993. Marine cyanophages infecting oceanic and coastal strains of Synechococcus - abundance, morphology, cross-infectivity and growth-characteristics. Marine Ecology Progress Series, 92, 99-109.

Suttle, C.A. \& Chan, A.M., 1994. Dynamics and distribution of cyanophages and their effect on marine Synechococcus spp. Applied and Environmental Microbiology, 60, 3167-3174.

Waterbury, J. \& Rippka, R., 1989. The order Chroococcales. In Bergey's Manual of Systematic Bacteriology (ed. J.T. Staley et al.), pp. 1728-1746. Baltimore, Williams \& Wilkins.

Waterbury, J.B. \& Valois, F.W., 1993. Resistance to cooccurring phages enables marine Synechococcus communities to coexist with cyanophages abundant in seawater. Applied and Environmental Microbiology, 59, 3393-3399.

Wilson, W.H., Joint, I.R., Carr, N.G. \& Mann, N.H., 1993. Isolation and molecular characterization of 5 marine cyanophages propagated on Synechococcus sp strain WH7803. Applied and Environmental Microbiology, 59, 3736-3743.

Wolfvecht, A., Paldor, N. \& Brenner, S., 1992. Hydrographic indications of advection convection effects in the Gulf of Elat. Deep-Sea Research Part A-Oceanographic Research Papers, 39, 13931401.

Wommack, K.E. \& Colwell, R.R., 2000. Virioplankton: viruses in aquatic ecosystems. Microbiology and Molecular Biology Reviewes, 64, 69-114.

Wyman, M., Gregory, R.P.F. \& Carr, N.G., 1985. Novel role of phycoerythrin in a marine cyanobacterium, Synechococcus strain DC2. Science, New York, 230, 818-820.

Submitted 14 December 2005. Accepted 22 February 2006. 